\title{
Italian standards for diabetes mellitus 2007: executive summary
}

\author{
Diabete Italia, AMD Associazione Medici Diabetologi, SID Società Italiana di \\ Diabetologia
}

\section{Project participants \\ Coordinating committee: \\ Graziella Bruno \\ Alberto De Micheli \\ Domenico Fedele \\ Luca Monge \\ Editors: \\ Matteo Bonomo \\ Agostino Consoli \\ Salvatore De Cosmo \\ Paolo Di Bartolo \\ Antonino Di Benedetto \\ Graziano Di Cianni \\ Francesco Dotta \\ Andrea Giaccari \\ Francesco Giorgino \\ Giorgio Grassi \\ Gabriella Gruden \\ Domenico Mannino \\ Danilo Orlandini \\ Maria Chantal Ponziani \\ Olga Vaccaro \\ Ester Vitacolonna}

\section{Advisors}

Method and EBM:

Antonio Nicolucci

A. De Micheli $(\bowtie)$

Unità Operativa Diabetologia, Azienda Ospedaliera

Universitaria San Martino, Largo Rosanna Benzi, 10,

16132 Genova, Italy

e-mail: alberto.demicheli@hsanmartino.it;

alberto_demicheli@tin.it

Quality issues/indicators:

Nino Cimino

Gualtiero de Bigontina

Illidio Meloncelli

Paediatric Diabetology:

Franco Meschi

Panel of judges:

Giovanni

Careddu

Giovanna

Cecchetto

Antonio Ceriello

Marco Comaschi

Giorgio Cruccu

Domenico

Cucinotta

Stefano Del Prato

Ottavio

Di Stefano

Giuseppe Fatati

Raffaele Foglia
Rosangela
Ghidelli

Raffaele Foglia

Ghidelli
GISED: Gruppo italiano di studio per l'educazione sul diabete Italian Study Group for Diabetes Education)

ANDID: Associazione nazionale dietisti (National Association of Dieticians)

AMD: Associazione medici diabetologi (Italian Association of Diabetologists)

AMD: Associazione medici diabetologi (Italian Association of Diabetologists) SIN: Società italiana di neurologia (Italian Society of Neurology)

AMD: Associazione medici diabetologi (Italian Association of Diabetologists)

SID: Società italiana di diabetologia (Italian Society of Diabetology)

Coordinator, The Ethics Committee, The Brescia Province's Doctors and Dentists Association

Associazione italiana di dietetica $e$ nutrizione clinica (Italian Association of Dietetics and Clinical Nutrition) Counsellor to the Cassation Court OSDI: Associazione operatori sanitari di diabetologia italiani (Italian Diabetes Health Workers Association) 


\section{Carlo Giorda \\ Riccardo \\ Giorgino \\ Massimo Lepri \\ Renata Lorini}

Antonio Mafrici

Walter Marrocco

Gerardo Medea

Enrico Mongiovì

Michele Muggeo

Nicoletta

Musacchio

Gianfranco

Pagano

Maria Antonietta

Pellegrini

Paola Pisanti

Roberto

Pontremoli

Gabriele Riccardi

Simona Sappia

Giorgio Sesti

Roberto Sivieri

Angela Testi
AMD: Associazione medici diabetologi (Italian Association of Diabetologists)

SID: Società italiana di diabetologia (Italian Society of Diabetology)

Political and organisational issue expert SIEDP: Società italiana di endocrinologia e diabetologia pediatrica (Italian Society of Paediatric Endocrinology and Diabetology)

ANMCO: Associazione nazionale medici cardiologi ospedalieri (National Association of Hospital Cardiologists)

FIC: Federazione italiana di cardiologia (Italian Federation of Cardiology)

FIMMG/SIMEF: Federazione italiana medici di medicina generale/Società italiana medicina di famiglia (Italian Federation of General Practitioners/ Italian Society of Family Physicians)

SIMG: Società italiana di medicina generale (Italian Society of General Medicine)

SIPU: Società italiana di podologia universitaria (Italian Society of Podiatrists)

SID: Società italiana di diabetologia (Italian Society of Diabetology)

AMD: Associazione medici diabetologi (Italian Association of Diabetologists)

SID: Società italiana di diabetologia (Italian Society of Diabetology) AMD: Associazione medici diabetologi (Italian Association of Diabetologists) Planning General Directorate, The Ministry of Health

SIN: Società italiana di nefrologia (Italian Society of Nephrology)

SID: Società italiana di diabetologia (Italian Society of Diabetology)

CnAMC: Coordinamento nazionale delle associazioni di malati cronici-CittadinanAttiva (National Coordinating Board for Chronic Disease Patients' Associations. Active Citizenship Network)

SID: Società italiana di diabetologia (Italian Society of Diabetology)

Quality Issue Expert

Professor of Political Economics and Economics Applied to Social and Healthcare Service Production Sectors
Vincenzo

Trischitta

Giacomo

Vespasiani

SID: Società italiana di diabetologia (Italian Society of Diabetology)

AMD: Associazione medici diabetologi

(Italian Association of Diabetologists)

National steering committees:

AMD steering committee

President: $\quad$ Umberto Valentini

Vice President: Adolfo Arcangeli

Counsellors: $\quad$ Luciano Carboni, Gerardo Corigliano, Gualtiero De Bigontina, Alberto De Micheli, Rossella Iannarelli, Sergio Leotta, Alessandro Ozzello, Pietro Pata, Giovanni Perrone

Secretary: Antonino Cimino

Treasurer: $\quad$ Sandro Gentile

SID steering committee

President: $\quad$ Riccardo Vigneri

Elected Paolo Cavallo Perin

President:

Counsellors: Graziella Bruno, Brunella Capaldo, Mauro Cignarelli, Paola Fioretto, Simona Frontoni, Giulio Marchesini Reggiani, Piero Marchetti, Emanuela Orsi, Paolo Sbraccia, Bruno Solerte, Giancarlo Tonolo

Secretary: Lucia Frittitta

Treasurer: Pierpaolo De Feo

Regional presidents:

AMD: Antimo Aiello, Giuseppe Armentano, Vincenzo Armentano, Francesco Calcaterra, Salvatore De Cosmo, Paolo Di Bartolo, Antonino Di Benedetto, Giovanni Divizia, Paolo Foglini, Giampaolo Magro, Giulio Mariani, Gisella Meloni, Mario Pupillo, Mauro Rossi, Roberto Sturaro, Concetta Suraci, Angelo Venezia, Giorgio Zanette.

SID: Alberto Bruno, Fabio Capani, Giovanni Corsini, Carla Giordano, Francesco Giorgino, Annunziata Lapolla, Silvana Manfrini, Domenico Mannino, Edoardo Mannucci, Anna Maria Angela Marinaro, Nazario Melchionda, Roberto Morea, Giuseppe Pugliese, Teresa Salvatore, Luciano Scionti, Claudio Taboga, Roberto Trevisan.

Acknowledgements for critical contributions:

SID Study Groups, AMD Study Groups, SID, AMD and Diabete Italia Interassociation Groups; Mariano Agrusta, Angelo Avogaro, Enzo Bonora, Emanuele Bosi, Paolo Brunetti, Daniela Bruttomesso, Tiziana Bufacchi, Franco Burla, Anna Cantagallo, Carlo Caravaggi, Anna Ciardullo, 
Marco Comoglio, Renzo Cordera, Carlo Coscelli, Gaetano Crepaldi, Paolo Di Berardino, Sergio Di Pietro, Katherine Esposito, Ezio Faglia, Danila Fava, Massimo Federici, Francesco Galeone, Aldo Galluzzo, Stefano Genovese, Luigi Gentile, Sandro Gentile, Paolo Gentili, Annalisa Giancaterini, Marta Letizia Hribal, Renato Lauro, Davide Lauro, Luigi Laviola, Luca Lione, Stanislao Lostia, Giuseppe Marelli, Massimo Massi Benedetti, Roberto Mingardi, Valerio Miselli, Claudio Noacco, Massimo Orrasch, Giuseppe Paolisso, Gabriele Perriello, Gianluca Perseghin, Piermarco Piatti, Giuseppe Pipicelli, Andrea Pizzini, Antonio Ettore Maria Pontiroli, Massimo Porta, Guido Pozza, Paolo Pozzilli, Francesco Purrello, Donata Richini, Angela Rivellese, Carlo Maria Rotella, Giuseppe Sanfilippo, Alessandro Sergi, Anna Solini, Marco Songini, Vincenza Spallone, Sebastiano Squatrito, Paolo Tessari, Antonio Tiengo, Laura Tonutti, Mariella Trovati, Natalia Visalli, Ivana Zavaroni.

\section{Introduction}

The Italian Standards for Diabetes Mellitus proposed herein have been drafted by two Italian scientific diabetes societies (AMD and SID) to provide clinicians, patients, researchers and those involved in diabetes care with recommendations for the diagnosis and management of diabetes and its complications. They also propose treatment goals-substantiated by extensive scientific evidence- on which therapeutic decisions can be based and, treatment quality evaluation tools adapted to the Italian framework. They are the scientific reference model for diabetes care, both concerning goals and processes. The project proposes to share common treatment models and goals for the care of diabetic patients in our practical national framework with Italian diabetologists and all medical and non medical professionals involved in diabetes care. Italian Standards for Diabetes Mellitus can be deemed as a scientific landmark for integrated management, disease management, professional accreditation and hospitals' daily need to create effective and efficient diagnostic and care pathways.

The level of scientific tests behind every recommendation has been classified, as envisaged by the National Plan for Guidelines (http://www.pnlg.it) (Table 1). The document enlarges on "desirable" goals in the management of most diabetics; individual preferences, comorbidity and other factors related to the individual patient can, however, justify the various decisions. Moreover, the Standards are not designed to prevent either further diagnostic investigations or patient management by other specialists, when required. For detailed information, please refer both to the mentioned guidelines and to references listed in each chapter.

\section{Methodology}

There are many international guidelines for diabetes mellitus: specifically, Standards of Medical Care published by the American Diabetes Association (ADA) has long been a landmark for diabetologists due to its pragmatic features, systematic updates and recommendations furnished with evidence levels.

However, not always can treatment standards, which suit other populations and social and healthcare situations, be applied to the Italian framework; moreover, there are certain divergent views in the international diabetological community and a national stand concerning the clinical application of these points is required.

On the basis of indications provided by the International Diabetes Federation (The IDF does not recommend 'reinventing the wheel', but does strongly encourage the redesign of the wheel to suit local circumstances), derived guidelines have thus been drafted for obvious reasons related to the rational use of both human and economic resources. Furnished with levels of evidence and recommendations, they are based on the critical evaluation of the ADA's original 2006 paper, other international guidelines and, when necessary, the primary sources available in literature, adapting them and targeting them at the Italian framework. Moreover, the paper integrates previously existing Italian guidelines, data and notes on the specific Italian situation and aspects that are not developed in the ADA's paper. Process and outcome indicators have been added to the recommendations whenever possible- they have already been tested in the AMD data file-to provide assessment tools.

The Consensus Conference Method, which requires a jury to discuss and evaluate a proposal presented by a team of experts appointed by both AMD and SID, was chosen to reach the paper's final draft.

\section{The process}

The process that led to these Italian Standards for Diabetes Mellitus is briefly described below.

- The project was commissioned by AMD and SID's National Steering Committees with Diabete Italia's approval. They requested a technical document drafted by experts and discussed by a jury, which they could approve as an official document on the views of scientific societies.

- The Editorial Team, which numbered 20 diabetologists with a Coordinating Committee of four diabetologists, edited the draft of the text's specific topics. The Editorial Team resorted to the contribution of expert 
Table 1 Evidence levels and recommendation strength

Evidence levels

Evidence

types

I

Evidence obtained from many controlled randomised clinical trials and/or from systematic reviews of randomised trials.

II Evidence obtained from one randomised trial with an appropriate pattern.

III Evidence obtained from non-randomised cohort studies with either concurrent or historical controls or their metanalyses.

IV Evidence obtained from either retrospective casecontrol studies or their metanalyses.

V Evidence obtained from case studies ("series of cases") without a control group.

VI Evidence based on the opinions of either authoritative experts or expert committees, as specified in both the guidelines and consensus conference, or based on the opinions of team members that drafted these guidelines.

Recommendation strength

\section{Strength}

A

The performance of a special procedure or diagnostic investigation is highly recommended. This strength indicates a special recommendation backed by scientific evidence of good quality, though not necessarily type I or II.

B

It is based on the doubt that the special procedure or intervention in question must always be recommended, but it is deemed that its performance must be carefully considered.

C There is a basic uncertainty either for or against the recommendation to perform the procedure or intervention.

D The procedure's performance is not recommended.

E There are strong recommendations against the procedure.

consultants in methodology, EBM and quality issues and of a consultant paediatric diabetologist recommended by the Italian Society of Paediatric Endocrinology and Diabetology.

- A highly interdisciplinary jury numbering diabetologists and members of other healthcare professions dedicated to diabetes care and lay members was created to guarantee the paper's best applicative efficacy. It counted seven diabetologists appointed by the AMD, seven diabetologists appointed by the SID, one dietician, one neurologist, one nephrologist, one cardiologist, one paediatrician, two general practitioners, one expert in therapeutic education, one nurse, one podiatrist, one dietician, one lawyer, one expert in bioethics, one representative of the Ministry of Health, one epidemiologist, one expert in healthcare economics, one member of the Tribunal of Patients' Rights, one quality expert and, one expert in political-organisational issues.

- The Jury received the document's preliminary text and heard the presentations of single topics and some queries on the document's controversial aspects at the Consensus meeting held in Frascati on 8-9 November 2006. The meeting was open to both National Steering Committees and to Presidents of the AMD and the SID's regional branches. The Jury later met behind closed doors to analytically assess the document and a final plenary session presented and motivated criticisms, remarks and proposals in view of the document's first review.

- On the basis of these conclusions, the Editorial Team edited a second version of the document. The critical contribution of experts and leaders of AMD and SID study groups was requested during this reviewing phase.

- The Jury's reappraisal of the paper's second version enabled the Coordinating Committee to draft the final paper, whose version presented herein was finally approved by the AMD and the SID's National Steering Committees.

The names of all participants are listed above.

The paper will be published in this hardcopy version and in a pocket edition, besides an electronic version for the Web and palmtop PCs.

Regional initiatives focused on circulating, sharing and systematically implementing Italian Standards for Diabetes mellitus and inserted in the framework and goals of ministerial initiatives for the organisation of diabetes care and for integrated management with general medicine are scheduled to be held in 2007.

As with all guidelines, the document will be periodically updated by a Committee that is specially appointed for this purpose.

In the Executive Summary we included the graded recommendations only: for more detailed information refer to the full document. [http://www.aemmedi.it/, http://www. siditalia.it/]

\section{Diagnostic criteria and classification}

Diabetes is diagnosed if:

- Fasting blood glucose is $\geq 126 \mathrm{mg} / \mathrm{dl}$ (after at least an 8 h fasting);

or

- Random blood glucose is $\geq 200 \mathrm{mg} / \mathrm{dl}$ (irrespective of the intake of food); 
or

- Blood glucose is $\geq 200 \mathrm{mg} / \mathrm{dl} 2 \mathrm{~h}$ after a $75 \mathrm{~g}$ oral glucose load. (VI, B)

Blood glucose testing for diagnostic and screening purposes must be performed on venous plasma and confirmed in two different tests, if abnormal; the use of glucometers is not recommended as testing modes are hard to standardise. (VI, B)

The following tests are not required for diabetes diagnosis:

- HbA1c;

- basal insulinemia and during OGTT insulinemia ;

- postprandial glucose and diurnal glycemic profile. (VI, E)

Blood glucose alterations that are not diagnostic for diabetes must be classified as:

- Impaired fasting glucose (IFG) (fasting blood glucose: $100-125 \mathrm{mg} / \mathrm{dl})$;

- Impaired glucose tolerance (IGT) (blood glucose concentration $2 \mathrm{~h}$ after an oral glucose load $\geq 140$ and $<200 \mathrm{mg} / \mathrm{dl}$ ). (VI, B)

In subjects with IFG and IGT all cardiovascular risk factors must be examined to plan an appropriate care pathway. (VI, B)

In subjects with IFG and either abdominal obesity or metabolic syndrome it could be useful to perform an OGTT for better diagnostic and prognostic definition. (VI, C)

\section{Screening for type 2 diabetes}

Screening programmes are recommended for people at high risk of diabetes (selective screening, Table 2) identified during a medical check up (opportunistic screening). (VI, B)

Screening programmes based on Fasting Blood Glucose have a more favourable cost/efficacy ratio than those based on OGTT. (VI, B)

An OGTT can be performed in high-risk subjects to better define the individual risk of diabetes and cardiovascular diseases. (VI, B)

In case of normal screening tests, high risk subjects should be re-examined after 2-3 years; moreover, they must be provided with useful instructions to both change their lifestyle and to reduce diabetes risk factors. (VI, B)

\section{Screening and diagnosis of gestational diabetes}

Risk of gestational diabetes must be assessed during the initial evaluation of a pregnant woman (Table 3): an OGTT
Table 2 Subjects with a high risk of diabetes

IFG, IGT or past gestational diabetes

Age $\geq 45$ years, especially with $\mathrm{BMI} \geq 25 \mathrm{~kg} / \mathrm{m}^{2}$ or abdominal obesity

Age $<45$ years, overweight $\left(\mathrm{BMI} \geq 25 \mathrm{~kg} / \mathrm{m}^{2}\right.$ ) and one or more of the following conditions:

First degree relative with type 2 diabetes;

Members of a high risk ethnic group;

Arterial hypertension $(\geq 140 / 90 \mathrm{mmHg}$ ) and/or antihypertensive treatment;

Low levels of HDL cholesterol ( $\leq 35 \mathrm{mg} / \mathrm{dl})$ and/or high triglyceride concentration ( $\geq 250 \mathrm{mg} / \mathrm{dl})$;

Clinical evidence of cardiovascular diseases;

Low level of physical activity;

Polycystic ovarian syndrome or other insulin-resistant conditions like Acanthosis nigricans;

Women who delivered a baby weighting $>4 \mathrm{~kg}$.

Children aged $>10$ years, with BMI $>85$ th percentile and two of the following conditions:

First or second degree relative with type 2 diabetes;

Mother with gestational diabetes;

Signs of insulin-resistance or associated conditions (hypertension, dyslipidemia, acanthosis nigricans, polycistic ovarian syndrome);

Members of a high-risk ethnic group.

Table 3 Risk profiles

Low risk. Screening is not required when all the following characteristics are present:

Age $<25$ years;

Normal pre-pregnancy weight;

Negative family history for diabetes mellitus;

Negative case history for impaired glucose tolerance;

Obstetric history with no unfavourable outcome;

Ethnic group with a low prevalence of gestational diabetes.

Medium risk. Women with intermediate characteristics between low and high risk: the glucose tolerance test should be performed between the 24th and 28th week.

High risk. Screening must be performed as early as possible (and repeated between the 24th and 28th week of gestation, if the first test is normal), if one or more of the following characteristics is present:

Positive family history for diabetes in first degree relatives;

Previous diagnosis of impaired glucose tolerance;

Foetal macrosomia during past pregnancies;

Obesity (BMI $>30 \mathrm{~kg} / \mathrm{m}^{2}$ );

Pronounced glycosuria during the current pregnancy.

must be performed at the 24th-28th week of gestation when there is an intermediate risk; if the risk is high, the test must be performed as early as possible. $(\mathbf{V}, \mathbf{B})$

The screening procedure suggested is the two-phase oral glucose load test $(50 \mathrm{~g}$ glucose challenge test) and diagnostic test with $100 \mathrm{~g}$ of glucose (VI, B) 
Glucose tolerance should be reassessed in all women with gestational diabetes 6 weeks after delivery with an OGTT (VI, B)

\section{Primary prevention of type 2 diabetes}

Avoiding overweight and performing regular physical exercise (20-30 min a day or 150 min a week) is the most appropriate way of reducing the risk of type 2 diabetes mellitus in subjects with IGT. (I, A)

Subjects with IGT must be given counselling concerning weight loss and indications to increase physical exercise. (I, A)

Subjects with IGT must be encouraged to change their diet habits: reduce the total intake of fat $(<30 \%$ of the daily energy intake) and especially of saturated fatty acids (less than $10 \%$ of the daily calorie intake); increase the intake of vegetable fibre (at least $15 \mathrm{~g} / 1,000 \mathrm{kcal})$. (I, A)

Pharmacological treatment, though less effective, can be considered for obese subjects with IGT in whom the lifestyle interventions have either failed or cannot be applied. (I, B)

When other strategies have proved ineffective, bariatric surgery can be considered a therapeutic option allowing to prevent the onset of type 2 diabetes in subjects with severe obesity and IGT. (I, C)

\section{Diabetes care}

Diabetics must be cared for by both the general practitioner and the diabetes team, which, coordinated by a diabetologist, comprises doctors, nurses, dieticians, podiatrists and mental health professionals skilled in implementing an integrated disease management approach designed to treat a chronic disease. (VI, B)

Diabetics must actively participate in the care plan, which is drafted like a personalised therapeutic alliance between the patients, his/her family and diabetes team members. The patient's age, education and employment, physical exercise practised, diet habits, social and economic conditions, personality, cultural factors and the presence of other diseases or complications of diabetes will be focused. (VI, B)

The care plan must comprise an education program centred on self-management of diabetes to provide-by applying diversified strategies and techniques to suit the patient's age, social status and cultural level-appropriate knowledge on problem-solving methods applied to disease management. The care plan implementation requires every aspect to be clarified and agreed on between the patient and the diabetes team, besides the definition of achievable goals. (VI, B)

Initial evaluation (Table 4)

A diabetic patient's first evaluation envisages a complete medical check up that also seeks chronic complications typical of the disease and laboratory tests to define the patient's general clinical conditions. If diabetes had already been diagnosed, the treatment established and the degree of glycemic control obtained must be reassessed and the disease management plan must be redrafted, if necessary, once key points have been defined. (VI, B)

Glycemic control

\section{Evaluating glycemic control}

(a) Glycated haemoglobin (HbA1c)

- Evaluation of glycemic control achieved by a diabetic envisages periodical $\mathrm{HbAlc}$ testing. (VI, B)

- HbA1c dosage must be tested at least twice a year in all diabetics, even when glycemic control is stable within the therapeutic goal. (VI, B)

- HbA1c dosage must be tested every 3 months in patients, whose hypoglycemic treatment has been changed or whose therapeutic goal has either not been reached or is not stable in time. (VI, B)

(b) Self-monitoring of blood glucose

- Blood glucose self-monitoring with the diabetes team check is an essential factor in the self management of diabetes both to achieve the therapeutic goals and to reduce the risk of acute hypoglycaemia. (VI, B)

- Daily self-monitoring (at least 3-4 checks/day) is essential for type 1 diabetics under intensive insulin therapy. (II, A)

- Blood glucose self-monitoring-by varying frequency and testing modes-is useful for type 2 diabetics under insulin therapy. (III, B)

- Discontinuous blood glucose self-monitoring is potentially useful for type 2 diabetics on either oral or diet therapy, but no clear evidence is available concerning its efficacy on glycemic control. (VI, C)

- Self-monitoring of post-prandial blood glucose can be useful to both obtain good glycemic control and to achieve the target post-prandial blood glucose concentration. (VI, B)

- Self-monitoring frequency must be adjusted to intercurrent events and increased in clinical conditions, such as an intercurrent disease, unnoticed episodes of 
Table 4 The diabetic patient initial evaluation

Family history

Family history of diabetes, arterial hypertension, dyslipidemia, cardiovascular diseases and other endocrine diseases.

Physiological history

Physical exercise practised.

Lifestyle and cultural, psychosocial, educational and economic factors that can influence diabetes management.

Use of tobacco, alcohol and narcotics.

Evaluate diet habits, nutritional condition, weight history, growth and development in children and adolescents.

Contraception, sexual history and reproduction.

Past medical history

History and treatment of other diseases, including endocrine ones and eating disorders.

Cardiovascular risk factors: smoking, hypertension, obesity and dyslipidemia.

Past $\mathrm{HbA1c}$ values.

Frequency, severity and causes of acute complications (i.e. ketoacidosis and hypoglycemia).

Evaluate in detail past therapeutic programmes, prescribed diet, extent of education on the self management of diabetes and approach to the disease.

Recent medical history

Symptoms related to the diagnosis of diabetes.

Symptoms related to diseases that can cause secondary diabetes (i.e. haemochromatosis, pancreatic diseases).

Current diabetes treatment: drugs, diet plan, self-monitoring.

Past and current infections of skin, feet, teeth or the genitourinary system.

Symptoms of diabetes complications in eyes, kidneys, peripheral nerves, genitourinary system (including sexual diseases), bladder and gastrointestinal function (including celiac disease in type 1 diabetes), heart, cardiovascular system and feet, and current treatment

Use of drugs that can interfere with blood glucose concentrations

Evaluate mood disorders.

Physical examination

Height and weight (related to normal age parameters in children and adolescents).

Abdominal circumference.

Sexual maturation (if in peripuberty).

Lying and standing blood pressure (compare with normal age parameters in children and adolescents)

Ophthalmological examination of fundus oculi.

Examination of the oral cavity.

Palpation of the thyroid.

Heart and lung semiotics.

Abdominal palpation (to highlight hepatomegaly).

Palpation and auscultation to evaluate pulses and detect any vascular murmurs.

Evaluate hands.

Examine feet.

Examine the skin (especially insulin injection sites).
Table 4 continued

Neurological examination.

Laboratory tests

HbA1c.

Fasting lipid profile, comprising total cholesterol, HDL cholesterol, triglycerides and LDL cholesterol.

Liver function test and further investigations for suspected liver steatosis.

Test microalbuminuria in all type 2 diabetics and in type 1 diabetics with disease duration $>5$ years.

Serum creatinine and estimated glomerular filtration.

On diagnosis of type 1 diabetics: screen for autoimmune thyroiditis and celiac disease: TSH, FT4, anti-thyroid antibodies, EMA or antitransglutaminase ${ }^{\mathrm{a}}$ and $\operatorname{IgA}$.

Urine tests to evaluate ketonuria, proteinuria and urinary sediment.

Diagnostic investigations and specialist consultations

ECG in the adult, if clinically required.

Ophthalmological examination, if required.

Family planning for women of childbearing age.

Nutritional Medical Treatment, if required.

Specialist consultation on educational therapy, if it is not provided by either the doctor or other diabetes team members.

Specialist consultation on behavioural therapy, if required.

Specialist consultation for feet, if required.

Other specialist consultations and services, if required.

${ }^{\text {a }}$ If normal, yearly check TSH, antithyroid antibodies, EMA or antitransglutaminase in paediatric patients. If EMA or antitransglutaminase are positive on 2 occasions, perform an intestinal biopsy to confirm the diagnosis of celiac disease with a histological examination

hypoglycaemia, hypoglycaemia at night and changes in hypoglycemic treatment. (VI, B)

- The patient must be taught to self-monitor blood glucose; moreover, glucometer accuracy must be periodically checked and treatment must be adjusted to suit measured values, even resorting to a commonly agreed algorithm. (VI, B)

- Patient training to self-monitor blood glucose must be added to an educational programme that is both conducted and controlled in the middle and long term by nursing personnel experienced in the field of diabetes. (VI, B)

\section{Glycemic goals}

Diabetes treatment must be speedily adjusted in every patient to obtain near normal blood glucose concentrations and stable $\mathrm{HbA1c}$ values under 7\% (Table 5). These values will enable prevention of both the incidence and progression of micro- and macro-vascular complications. (I, A)

Lower glycemic control goals (HbA1c $<6.5 \%)$ can be considered for individual patients. (III, B) 
Table 5 Glycemic goals in type 1 and 2 diabetic adults

$\mathrm{HbA1c}<7.0 \%^{\mathrm{a}}(<6.5 \%$ in individual patients)

Fasting and pre-prandial blood glucose $90-130 \mathrm{mg} / \mathrm{dl}^{\mathrm{c}}$

Post-prandial blood glucose ${ }^{\mathrm{b}}<180 \mathrm{mg} / \mathrm{dl}^{\circ}$

${ }^{a}$ Referring to $4.0-6.0 \%$ in the non diabetic population with the method adopted by the DCCT study

b Post-prandial blood glucose must be tested $2 \mathrm{~h}$ after the meal's start

${ }^{c}$ Fasting blood glucose $<110 \mathrm{mg} / \mathrm{dl}$ and post-prandial blood glucose $<145 \mathrm{mg} / \mathrm{dl}$ must be pursued in type 2 diabetes (IDF 2005)

Slightly higher glycemic control can be considered in both small children and patients with acute episodes of hypoglycaemia, reduced life expectancy or comorbid conditions. (VI, B)

Algorithms for insulin therapy are recommended for Intensive Care patients as they facilitate the achievement of glycemic goals. (II, B)

\section{Medical nutrition therapy}

\section{General recommendations}

To achieve therapeutic goals, people with diabetes or impaired glucose tolerance must be administered personalised medical nutrition therapy by a dietician, preferably a diabetes team member trained in the diabetes field. (III, B)

Nutrition consultation must consider people with IGT or diabetes mellitus' personal requirements and intention to change. (VI, B)

Vegetables, beans, fruit and cereal typical of the Mediterranean diet must be added to the diet of people with type 1 and 2 diabetes mellitus. The intake of fibre rich food with a low Glycemic Index must be encouraged especially when a diet is rich in carbohydrates. (I, A)

There is no evidence to recommend the use of "diet" foods for diabetics. (VI, D)

\section{Overweight and obesity}

Weight loss is recommended in all overweight (BMI 25.0$\left.29.9 \mathrm{~kg} / \mathrm{m}^{2}\right)$ or obese $\left(\geq 30.0 \mathrm{~kg} / \mathrm{m}^{2}\right)$ adults. (I, A)

The main approach to weight loss is a change in lifestyle, which involves reducing calorie intake and increasing physical exercise. A moderate reduction in calorie intake (300-500 kcal/day) and a moderate increase in energy consumption (200-300 kcal/day) ensure slow but progressive weight loss $(0.45-0.90 \mathrm{~kg} /$ week). (I, A)

Moderate physical exercise must be recommended to suit the patient's inclination and capacity when the program starts. This exercise must then gradually increase in duration and frequency to $30-45 \mathrm{~min}$ a day of moderate aerobic exercise for 3-5 days a week (goal: $150 \mathrm{~min} /$ week). Higher levels of physical exercise-at least $1 \mathrm{~h} \mathrm{a}$ day of moderate activity (walking) or $30 \mathrm{~min}$ a day of more vigorous exercise (jogging) — can be required to obtain effective long-term weight loss. (VI, B)

\section{Carbohydrates}

The daily intake of carbohydrates with the diet must provide $45-60 \%$ of total daily calories. (VI, C)

Considering these limits, the patient's metabolic features will suggest the most appropriate intake for type 1 and 2 diabetics. (I, A)

Diets with a low-carbohydrate content (specifically reduced below $130 \mathrm{~g} /$ day) are not recommended for diabetics. (III, B)

Both the quantity and quality of carbohydrates in food can influence the glycemic response. Controlling total carbohydrate intake with either an exchange diet or the carbohydrate count is a key strategy to obtain glycemic control in patients under insulin therapy with a daily multidose pattern (basal-bolus). (I, A)

Assessing the quantity, quality and distribution of the daily intake of carbohydrates can facilitate achieving optimal glycemic control. All patients treated with hypoglycemic drugs, especially with insulin therapy, require the time of intake and drug dosage to be evaluated and adapted to the quantity and nature of carbohydrates taken. (III, B)

\section{Sucrose}

As with the population at large, in diabetics too the total intake of sucrose should not exceed $10 \%$ of the total energy daily introduced with food. A more restrictive attitude can be useful for people who need to lose weight. (I, A)

\section{Glycemic index}

The Glycemic index can be a useful indicator in the choice of food rich in carbohydrates to be added to the diet of diabetics. (III, B)

\section{Fibre}

People with type 1 and 2 diabetes must be encouraged to introduce food with high-fibre content. (I, A)

The ideal fibre intake with the diet should be more than $40 \mathrm{~g} / \mathrm{day}$ (or $20 \mathrm{~g} / 1,000 \mathrm{kcal} / \mathrm{day}$ ); most of it should be soluble. (I, A)

Daily consumption of five portions of either vegetables or fruit and a weekly intake of four portions of beans can meet minimum fibre requirements. (III, B) 


\section{Proteins}

Individuals with any degree of chronic renal failure must limit protein intake to the recommended dose $(0.6-0.8 \mathrm{~g} / \mathrm{kg})$ to reduce the risk of nephropathy developing any further. (I, A)

In patients with no history of nephropathy, the protein intake should provide $10-20 \%$ of the total energy daily supplied by food. (VI, B)

\section{Fat}

Fat intake must not contribute over $30 \%$ of the total energy daily supplied by food. (III, B)

The daily intake of saturated fat must be less than $10 \%$ of total calories. A lesser amount $(<7 \%)$ can be useful if LDL cholesterol is $>100 \mathrm{mg} / \mathrm{dl}$. (I, A)

Oils rich in monounsaturated fatty acids (MUFA) are an important source of fat. Depending on the patient's preferences, they can provide 10-20\% of the total energy daily introduced with food. (III, B)

The intake of trans fatty acids must be minimised $(<1 \%)$. (VI, B)

Polyunsaturated fatty acids (PUFA) must not contribute over $10 \%$ of the total energy daily supplied by food. (III, B)

Cholesterol introduced with the diet must not exceed $300 \mathrm{mg} / \mathrm{day}$. It can be further reduced if LDL cholesterol is $>100 \mathrm{mg} / \mathrm{dl}$. (I, A)

In overweight patients, a fat intake below $30 \%$ of the total energy daily introduced can facilitate weight loss. (IV, C)

\section{Alcohol}

A moderate intake of alcohol (up to $10 \mathrm{~g} /$ day in women and $20 \mathrm{~g} /$ day in men) is acceptable, if the patient wishes to take alcoholic drinks. (III, B)

Patients treated with insulin may take alcohol only with meals comprising food containing carbohydrates to prevent the risk of dangerous prolonged episodes of hypoglycaemia. (VI, B)

\section{Sweeteners}

Calorie-free sweeteners (i.e. saccharin, aspartame, acesulfame-K, sucralose) are safe when taken in moderate daily doses. (I, A)

\section{Diet supplements}

Habitual intake of supplements, such as antioxidants (i.e. vitamin $\mathrm{E}, \mathrm{C}$ and $\beta$-carotene) is not recommended when there is no evidence of their long-term efficacy and safety. $(\mathbf{I}, \mathbf{D})$
The intake of food that is naturally rich in antioxidants, microelements and other vitamins must be encouraged. Hence diabetics must be encouraged to daily eating fruit and vegetables. (III, B)

Salt

Like the population at large, diabetics must be recommended to take less than $6 \mathrm{~g}$ /day of salt. (I, A)

\section{Specific nutritional interventions in type 1 diabetics}

Patients treated with either short acting insulin analogues or insulin pumps must adjust the pre-prandial insulin bolus to suit carbohydrates contained in meals. (I, A)

Patients treated with fixed doses of insulin must maintain carbohydrate intake at meals constant both concerning quantity and time. (III, B)

Pharmacological treatment

\section{Type 1 diabetes}

Keep close control of blood glucose to reduce the risk of chronic complications. (I, A)

The first choice treatment pattern is the basal-bolus. (IV, B)

The prescription of self-management algorithms for insulin therapy can facilitate the achievement of glycemic goals. (II, B)

When HbA1c values are higher than the goal, appropriate treatment variations should be speedily implemented to rapidly reach and maintain good glycemic control in time. (VI, B)

Take into account the patient's possible poor adhesion to the prescribed therapy. (I, A)

Treatment with insulin pumps should be prescribed to patients, whose educational evaluation and basal-bolus insulin therapy pattern (with rapid and slow acting analogues) have failed to achieve good metabolic control and, if there are frequent and/or asymptomatic hypoglycemic episodes. (I, B)

\section{Type 2 diabetes}

Continue close monitoring of blood glucose to reduce the risk of chronic complications. (I, A)

When HbAlc concentrations are higher than the glycemic goal, appropriate treatment variations must be speedily implemented to rapidly reach and maintain good blood glucose control in time. (VI, B)

The first choice drug is metformin when there is an overweight condition (BMI $>25 \mathrm{~kg} / \mathrm{m}^{2}$ ). (I, B) 
Secretagogues, metformin, glytazones and insulin are equally effective in reducing glycated haemoglobin. (I, A)

It is essential to combine two or more oral hypoglycemic drugs in patients who lack good control with monotherapy. (I, A)

It is essential to start either mono- or multi-injection insulin therapy when blood glucose control is inadequate even with multitherapy. (I, A)

Take into account possible poor adhesion to the prescribed treatment. (I, A)

Therapeutic education

People with diabetes must be instructed to self-manage their disease both at the time of diagnosis and later, to suit requirements. (III, B)

Education on the self-management of diabetes must be guaranteed by the team's healthcare personnel that are specially qualified with ongoing professional training to conduct educational activities. (VI, B)

All diabetes teams must have at least one healthcare operator who is specifically trained in therapeutic education. (VI, B)

When there is no educator, we encourage other team members to acquire this competence. (VI, B)

Education on the self-management of diabetes must also focus on psychosocial issues because emotional wellness is strongly associated with the positive outcome of treatment. (III, B)

Education on the self-management of diabetes must be appropriately acknowledged and paid as an integrated intervention system in the framework of NHS services. (VI, B)

\section{Physical exercise}

At least $150 \mathrm{~min} /$ week of moderately intense aerobic physical exercise $(50-70 \%$ of the maximum heart rate) and/or at least $90 \mathrm{~min} /$ week of intensive physical exercise ( $>70 \%$ of the maximum heart rate) are recommended to improve glycemic control, encourage maintenance of optimal body weight and reduce the risk of cardiovascular diseases. Physical exercise must be distributed in at least 3 days/week with intervals that do not exceed two consecutive days. (I, A)

Failing contraindications, type 2 diabetics must be encouraged to perform physical exercise against resistance thrice a week following a programme defined with the diabetologist and targeted at all major muscle groups. (I, A)

Introducing subjects who are out of training and have various degrees of relative sarcopoenia to a physical exercise programme-through gradual exercises against the resistance of small weights-can enable them to start aerobic exercises that strengthen muscles and increase aerobic capacity and weight loss. (VI, B)

The treadmill test is not recommended in asymptomatic subjects with a low risk of coronary heart disease who wish to start a physical exercise programme (risk of a cardiac event after 10 years $<10 \%$ ). (VI, D)

It is advisable to increase blood glucose self-monitoring before, during (exercise duration $>1 \mathrm{~h}$ ) and after physical exercise. Instructions must be provided concerning the need for carbohydrate integration and the management of hypoglycemic therapy. Physical exercise is not recommended with ketosis. Instructions must also be provided concerning the risk of hypoglycemic episodes during exercise and the risk of delayed hypoglycaemia after physical exercise. (VI, B)

Psychosocial evaluation applied to diabetes care

A preliminary evaluation of the psychological and social condition must be performed at the first examination and, anyhow, when adherence to the therapeutic regime is inadequate. (VI, B)

The psychosocial evaluation should study the attitude towards the disease and subsequent expectations, diabetesrelated complications, medical management and quality of life (both in a broad sense and related to the disease), economic, social and emotional resources and the patient's psychiatric history, if any. (VI, B)

Psychological treatment is better inserted in the framework of routine treatment than administered only when a specific problem or worsening in the psychic condition are detected. (VI, B)

Intercurrent diseases

When there is an intercurrent disease, pharmacological treatment must be reviewed and adjusted to suit either concomitant blood glucose alterations or the new risk profile induced by the disease in progress. (III, B)

\section{Hypoglycaemia}

Oral glucose $(15 \mathrm{~g})$ is the choice treatment for mild and moderate hypoglycaemia, though equivalent doses of any form of carbohydrates containing glucose can be used for this purpose; the effects of treatment should be evident within 15 min after ingestion. (VI, B)

The effect of treatment during a hypoglycemic condition can only be temporary. Hence, blood glucose must be tested every 15 min till at least two normal values are found without the administration of further treatment between two tests. (VI, B) 
The i.v. administration of hypertonic glucose solutions $(20-33 \%)$ is the choice treatment for acute hypoglycaemia when there is venous access, failing which, either intramuscular or subcutaneous administration of glucagon is recommended. (VI, B).

Glucagon must be available for all patients with a significant risk of acute hypoglycemic episodes (i.e. diabetics under insulin therapy who lack good glycemic control due to either unstable blood glucose concentrations or the onset of sudden hypoglycemic episodes with no forewarning). The administration of glucagon does not require the attendance of a healthcare professional. (VI, B)

\section{Vaccinations}

Yearly administer the anti-flu vaccine to diabetics aged over 6 months. (III, B)

Administer the pneumococcal vaccine to diabetic adults at least once in a lifetime. A single revaccination is recommended for subjects aged $>64$ years who had their first vaccination at least 5 years before. Other indications for revaccination are nephrotic syndrome, chronic renal failure and other immunocompromised conditions (i.e. organ transplant). (III, B)

\section{Diabetes managed care}

Achieving diabetes care goals envisages cooperation between the diabetes team, the general practitioner and, in a broad sense, the specialist in territorial medicine, in the framework of well defined care pathways based on an integrated multidisciplinary and multiprofessional approach that is implemented with the patient's informed consent. (III, B)

It is to be hoped that facilities directly involved in diabetes care have appropriate information systems with a special data storage system designed to store common basic data by format and plot to encourage data sharing and the definition and use of clinical indicators. (VI, B)

Either a specialised team or a diabetologist should be consulted when there is:

- newly diagnosed diabetes;

- insulin-treated diabetes;

- diabetes without good glycemic control;

- gestational diabetes either during pregnancy or in view of a pregnancy;

- diabetes developing either acute or chronic complications. (III, B)

\section{Prevention and management of diabetic complications}

\section{Cardiovascular diseases}

\section{Hypertension management}

Screening, diagnosis and monitoring Blood pressure must be tested at every visit. Systolic pressure $\geq 130$ $\mathrm{mmHg}$ or diastolic pressure $\geq 80 \mathrm{mmHg}$ must be confirmed on various days for 1 month. $(\mathbf{V}, \mathbf{B})$

Blood pressure must be checked every 3 months in patients under hypertensive treatment and every 4-8 weeks during the dose adjustment phase unless therapeutic goals are achieved. (VI, B)

Goals Antihypertensive treatment administered to diabetics focuses on achieving systolic pressure $<130 \mathrm{mmHg}$. (III, B)

Antihypertensive treatment administered to diabetics is designed to achieve diastolic pressure $<80 \mathrm{mmHg}$. (II, B)

Pressure goals $<125 / 75 \mathrm{mmHg}$ are recommended for diabetics with proteinuria $>1 \mathrm{~g} /$ day. (II, B)

Therapy Patients with either systolic pressure $=130$ $139 \mathrm{mmHg}$ or diastolic pressure $=80-89 \mathrm{mmHg}$ must change their lifestyle (i.e. reduce body weight if they are overweight, perform regular aerobic physical exercise, follow a low salt diet and reduce alcohol intake) and follow behavioural therapy for no more than 3 months. Start pharmacological treatment, if goals have not been achieved at the close of the said period. (VI, B)

Besides behavioural therapy and advice on lifestyle, hypertensive patients (systolic pressure $\geq 140 \mathrm{mmHg}$ or diastolic pressure $\geq 90 \mathrm{mmHg}$ ) must be administered intensive pharmacological therapy designed to achieve therapeutic goals. (I, A)

Frontline pharmacological treatment The choice of initial pharmacological treatment must consider comorbidities indicating either the use or exclusion of a certain drug category. (VI, B)

Patients with diabetic nephropathy (reduced estimated glomerular filtration rate or micro/macroalbuminuria) must be administered either ACE-inhibitors or ARBs, excepting pregnant women. (See diabetic nephropathy). (I, A)

Failing a comorbid condition, frontline pharmacological treatment for patients with blood pressure $>140 / 90 \mathrm{mmHg}$ must envisage a drug category that can reduce cardiovascular events in diabetic patients (i.e. ACE-inhibitors, ARBs, diuretics, calcium antagonists and beta-blockers). (I, A) 
Alphalytics are not recommended as frontline drugs for hypertension with diabetes. (I, E)

Treatment with ACE-inhibitors, ARBs or diuretics requires kidney function and serum potassium testing either 1-2 weeks after the treatment's start or after an increase in dosage; testing will then be performed either once a year or more frequently in patients with impaired kidney function. (VI, B)

Treatment with ACE-inhibitors and ARBs is contraindicated in pregnancy. (VI, E)

Standing blood pressure must be checked in people with diabetes and hypertension, when it is clinically indicated. (VI, B)

\section{Dyslipidemia management}

A complete lipid profile (total cholesterol, HDL and triglycerides) must be run at least yearly and at closer intervals in case of failure to achieve the therapeutic goal. (VI, B)

LDL cholesterol must be deemed the primary goal of treatment. (I, A)

Non-HDL cholesterol can be a secondary goal in diabetics with blood triglycerides $>200 \mathrm{mg} / \mathrm{dl}$. (III, B)

The apoB/apoA1 ratio can be a useful index of the cardiovascular risk in diabetics. (III, B)

Lifestyle changes (diet with low saturated fat and cholesterol and high fibre content, increased physical exercise) and corrections concerning cardiovascular risk factors (optimisation of glycemic control and pressure values, discontinuation of the smoking habit) are essential in dyslipidemic diabetics. (I, A)

Hypolipidemic treatment has proved effective as primary and secondary prevention in reducing cardiovascular risk (i.e. fatal and non fatal AMI and coronary revascularization) in type 2 diabetics. (I, A)

Statins are the first choice drugs in preventing cardiovascular diseases. (I, A)

Treatment with statins and lifestyle changes are recommended for LDL cholesterol $>130 \mathrm{mg} / \mathrm{dl}$ in diabetics aged $<40$ years with no additional cardiovascular risk factor. The therapeutic goal is to achieve LDL cholesterol $<100 \mathrm{mg} / \mathrm{dl}$. (V, B)

In high risk diabetics (i.e. one or more cardiovascular risk factors) hypolipidemic treatment must start irrespective of the LDL cholesterol level. The therapeutic goal is to achieve LDL cholesterol $<100 \mathrm{mg} / \mathrm{dl}$ (I, A).

LDL cholesterol $<70 \mathrm{mg} / \mathrm{dl}$ can be a therapeutic goal for diabetics with cardiovascular diseases and multiple cardiovascular risk factors that cannot be corrected. (VI, B)

Additional treatment goals can include achievement of plasma triglyceride levels $<150 \mathrm{mg} / \mathrm{dl}$ and HDL cholesterol $>40 \mathrm{mg} / \mathrm{dl}$ in men and $>50 \mathrm{mg} / \mathrm{dl}$ in women. (III, B)
Treatment with fibrates can be considered in diabetics with hypertriglyceridemia undergoing primary prevention treatment but with optimal LDL cholesterol levels. (II, B)

The combination statin + fibrate can be taken into account to achieve the therapeutic goal, but it is not supported by intervention studies targeted at diabetics. (VI, C)

\section{Use of antiplatelet drugs}

Antiplatelet treatment with acetylsalicylic acid is recommended for diabetics who have either experienced a past cardiovascular or cerebrovascular event or have peripheral arteriopathy. (I, B)

Antiplatelet treatment with acetylsalicylic acid is recommended for either diabetics aged over 40 years or those with at least one cardiovascular risk factor (i.e. hypertension, cigarette smoking, dyslipidemia, family history of cardiovascular events, microalbuminuria). (I, B)

Treatment with acetylsalicylic acid is best avoided in case of recent gastrointestinal bleeding, uncontrolled arterial hypertension, active liver disorders and drug allergies. (I, A)

The combination of acetylsalicylic acid + clopidogrel can be recommended for very high-risk diabetics (who have undergone revascularization surgery and have a history of past cerebral ischemic events or multiple vascular involvement). (II, B)

There are no controlled studies on the use of acetylsalicylic acid in diabetics aged under 30 years and in type 1 diabetics. (VI, C)

\section{Discontinuing the smoking habit}

Healthcare professionals must recommend all diabetics to discontinue cigarette smoking. (I, A)

Counselling on giving up smoking, nicotine replacement therapy and other drug treatment should be deemed as therapeutic components of diabetes care. (III, B)

\section{Coronary heart disease: screening and management}

Screening We recommend yearly screening for all diabetics from the time the disease is diagnosed. (III, B)

All diabetics, irrespective of the risk level, must yearly undergo:

- peripheral pulse examination and search for vascular bruits;

- basal ECG;

- Winsor index (if normal it can be evaluated again after 3-5 years). (VI, B) 
The following diagnostic investigations are also recommended for diabetics with a high cardiovascular risk (they must be repeated every 1-3 years, depending on the results obtained):

- carotid doppler ultrasound;

- doppler ultrasound of the lower limbs (with Winsor Index $<0.9$ or if arteries are not compressible)ischemia provocative tests (exercise ECG or exercise stress scintigraphy/echocardiography) (VI, B)

Treatment Diabetics with either a cardiovascular disease in progress or with high-risk factor levels must be administered appropriate treatment unless all altered values are normalised. (I, A)

Treatment with beta-blockers must be considered for diabetics with either past myocardial infarction or those who have undergone coronary revascularization surgery, irrespective of pressure values. (I, A)

Treatment with ACE-inhibitors to prevent cardiovascular events must be considered for diabetics aged over 55 years presenting at least one cardiovascular risk factor, irrespective of pressure values. (I, A)

Diabetic nephropathy: screening and management

\section{General recommendations}

Optimise glycemic control to reduce the risk and/or slow down the progress of nephropathy. (I, A)

Optimise pressure control to reduce the risk and/or slow down the progress of nephropathy. (I, A)

In patients with overt nephropathy, protein intake should be reduced to the recommended diet ration $(0.8 \mathrm{~g} / \mathrm{kg}$ $\left.\mathrm{day}^{-1}\right)$. A further reduction $\left(0.6-0.8 \mathrm{~g} / \mathrm{kg} \mathrm{day}^{-1}\right)$ can be useful in slowing down the drop in GFR in patients presenting the disease progress, despite optimised glycemic and pressure control and the use of ACE-inhibitors and/or ARBs. (III, B)

Correct any lipid profile alterations to both slow down the progress of nephropathy and reduce the associated cardiovascular risk. (I, B)

\section{Screening and stadiation}

Yearly test microalbuminuria in type 1 diabetics with a disease duration $>5$ years and in all type 2 diabetics, starting from the time of diagnosis and during pregnancy. (VI, B)

Serum creatinine should be yearly tested to estimate glomerular filtration rate in all adult diabetics, irrespective of the level of albumin excretion with urine. Serum creatinine alone should not be deemed as a kidney function marker; it must rather be used to estimate the glomerular filtration rate. (VI, B)

\section{Treatment}

ACE-inhibitors or ARBs should be recommended in the management of both microalbuminuria and macroalbuminuria, except during pregnancy. (I, A)

Despite the lack of appropriate direct comparisons between ACE-inhibitors and ARBs, some trials support each of the following statements:

- ACE-inhibitors slow down the progress of nephropathy in patients with type 1 diabetes, hypertension and any level of albuminuria. (I, A)

- ACE-inhibitors reduce the risk of microalbuminuria developing in patients with type 2 diabetes, hypertension and normal albuminuria. (I, A)

- ACE-inhibitors reduce the cardiovascular risk in patients with type 2 diabetes, normal blood pressure and microalbuminuria. (I, A)

- ACE-inhibitors and ARBs slow down the progress to macroalbuminuria in patients with type 2 diabetes, hypertension and microalbuminuria. (I, A)

- ARBs slow down the progress of nephropathy in patients with type 2 diabetes, hypertension, macroalbuminuria and renal failure (serum creatinine $>1.5 \mathrm{mg} /$ dl). (I, A)

- If one of the two drug categories is not tolerated, it should be replaced with the other. (VI, B)

Dihydropyridine calcium channel blockers (DCCB) as frontline treatment are not more effective than the placebo in slowing down the progress of nephropathy. Their use in nephropathy should be limited to the role of additional therapy to further reduce blood pressure in patients already treated with ACE-inhibitors or ARBs. (III, B)

The use of non-dihydropyridine calcium channel blockers (NDCCB), beta blockers and diuretics can be considered to control blood pressure in patients who do not tolerate ACE-inhibitors and/or ARBs. (VI, B)

When ACE-inhibitors, ARBs and diuretics are administered to patients with impaired renal function, we recommend testing renal function and serum potassium either 1-2 weeks after the treatment's start or after an increase in dosage and then at yearly or shorter intervals. (VI, B)

To evaluate both response to treatment and the progress of the disease, we recommend testing microalbuminuria/ proteinuria at 6-months intervals. (VI, B)

Consider a consultation with a doctor expert in diabetic nephropathy when GFR is $<60 \mathrm{ml} \mathrm{min}{ }^{-1} \times 1.73 \mathrm{~m}^{-2}$ or if there is any difficulty in managing either hypertension or hyperkalemia. A nephrology consultation is required when GFR is $<30 \mathrm{ml} \mathrm{min}^{-1} \times 1.73 \mathrm{~m}^{-2}$. (III, B) 
Diabetic retinopathy: screening and management

\section{General recommendations}

Optimising glycemic control reduces the risk and progress of retinopathy. (I, A)

Optimising pressure control reduces the risk and progress of retinopathy. (I, A)

Treatment with acetylsalicylic acid neither prevents diabetic retinopathy nor does it increase the risk of retinal bleeding. (I, A)

\section{Screening}

Adults with type 1 diabetes should first have their fundus oculi examined with pupil dilation by either an ophthalmologist or a trained health-care professional within 3-5 years after the onset of diabetes. (III, B)

Patients with type 2 diabetes should first have their fundus oculi examined with pupil dilation by either an ophthalmologist or a trained health-care professional soon after the diagnosis of diabetes. (III, B)

Subsequent testing in both types of diabetes should be repeated at least every 2 years by either a specialised ophthalmologist or a trained health-care professional expert in diagnosing and managing diabetic retinopathy. Less frequent testing can be considered if the ophthalmologist recommends it. The examination must be performed more frequently, if retinopathy progresses. (III, B)

Diabetic women who plan on starting a pregnancy should undergo a complete eye examination and be informed about the risk of diabetic retinopathy developing and progressing. (III, B)

Pregnant diabetic women should undergo a complete examination when pregnancy is confirmed and be monitored throughout pregnancy (at least every 3 months until delivery, failing eye lesions; when the ophthalmologist diagnoses retinopathy of any level, according to ophthalmologist judgement) and during the first year after delivery. (III, B)

Screening is not recommended in women with gestational diabetes mellitus because they do not have a high risk of developing diabetic retinopathy. (III, D)

Diabetic retinopathy can be screened with one or more of the following methods: ophthalmoscopy (direct and/or indirect) with dilated pupils and either colour or black and white photographs of the fundus oculi. $(\mathbf{V}, \mathbf{C})$

\section{Diagnosis}

Retinal fluorangiography is not recommended as a diagnostic tool for diabetic retinopathy. (VI, D)
Retinal fluorangiography must be performed in view of laser treatment in all cases, whose lesions require a pathogenetic interpretation that cannot be provided on the basis of the clinical examination. Specifically:

- pathogenetic interpretation of macular edema;

- detection of suspicious neovascularizations;

- exact definition of ischemic retinal areas;

- study of the macula in cases of clinically unjustified impaired vision. (VI, B)

Refer to the ophthalmologist in case of:

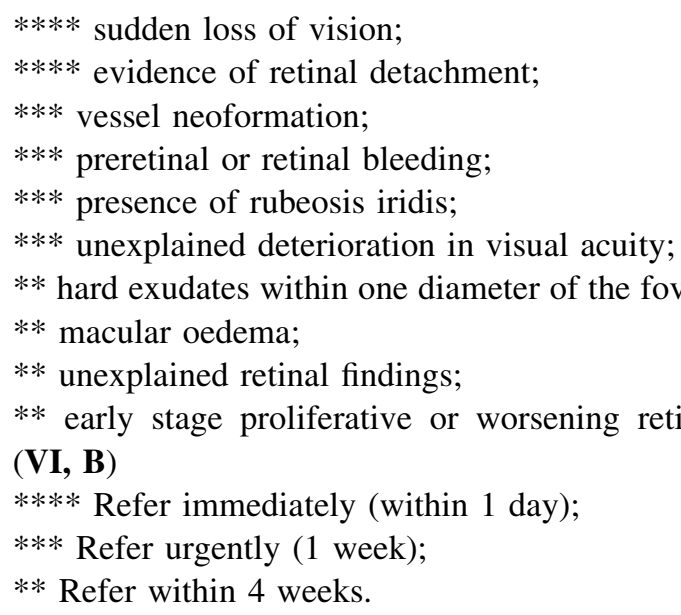

\section{Treatment}

Laser treatment reduces the risk of impaired vision in patients with high-risk retinal lesions. (I, A)

Promptly refer patients with any level of macular oedema, severe non proliferative diabetic retinopathy (NPDR) or any proliferative diabetic retinopathy (PDR) to an ophthalmologist who is knowledgeable and experienced in the management and treatment of diabetic retinopathy. (I, A)

Patients with macular oedema, severe NPDR or PDR require a prompt consultation with an ophthalmologist who is knowledgeable and experienced in the management and treatment of diabetic retinopathy because laser treatment, especially at this stage, is associated with either a $50 \%$ reduction in the risk of acute loss of sight or the need for vitrectomy, especially in patients with type 2 diabetes mellitus and severe NPDR. (VI, B)

Patients with serious sight impairment should be referred to rehabilitation. $(\mathbf{V}, \mathbf{B})$

\section{Follow-up}

Testing frequency must be: every 2 years, if there is no diabetic retinopathy; yearly, if there is mild to moderate background diabetic retinopathy (VI, B); earlier 
(3-6 months), if either new lesions are detected or if lesions have worsened, compared to the last follow up, if there is exudate within one diameter of the foveal disk, if the patient has a high risk of progress (rapid improvement in glycemic control, presence of arterial hypertension or renal complications). (VI, B)

Diabetic neuropathy: screening and management

\section{General recommendations}

Optimising glycemic control reduces the risk of the onset and progress of neuropathy. (I, A)

Screening for neuropathy must be performed on all type 2 diabetics at the time of diagnosis and on type 1 diabetics after 5 years of disease duration. Subsequent testing must be performed at yearly intervals. (VI, B)

\section{Distal symmetrical polyneuropathy}

Screening for chronic distal symmetrical sensitive and motor polyneuropathy must be implemented with simple clinical tests, such as pressure sensitivity testing with the $10 \mathrm{~g}$ single filament and vibratory sensibility testing on the big toe with a tuning fork, preferably performed within a structured score-based system. (I, A)

Screening for distal symmetrical polyneuropathy does not require electrophysiological testing, which is instead useful towards a differential diagnosis, when clinical characteristics are atypical. (VI, B)

If distal symmetrical polyneuropathy is diagnosed, the diabetic should be inserted in a specific educational programme to prevent ulceration and amputation of diabetic feet. (III, B)

\section{Vegetative neuropathy}

Screening for vegetative neuropathy must be performed with a detailed case history integrated by cardiovascular reflex testing, since symptoms are non specific and do not allow a diagnosis of vegetative dysfunction. (VI, B)

Cardiovascular tests are very useful when symptoms suggest vegetative dysfunction, when there is a high cardiovascular risk or a risk of microangiopathic complications (diabetic retinopathy or nephropathy), before major surgery, when planning a physical exercise programme and, in diabetics with poor glycemic control. (VI, B)

\section{Pharmacological treatment}

Pain generated by peripheral neuropathy can be relieved by anticonvulsants and serotoninergic, noradrenergic and tricyclic antidepressants (first choice drugs) and opiates (second choice drugs). (I, A)

Erectile dysfunction

Erectile dysfunction (ED) is an endothelial dysfunction equivalent; hence, it implies a strong risk of atherosclerotic complications. This involves the need for cardiovascular diagnostic investigations. (I, A)

The presence of ED in type 2 diabetics must be sought at the time of diagnosis and, evaluated again once a year. ED must be sought in type 1 diabetics with a long-disease duration ( $>10$ years) or with chronic complications, especially neuropathy and vasculopathy. (VI, B)

Screening, which must be periodically performed, simply consists in asking: "Over the past 6 months have you noticed any significant changes during sexual intercourse?" (VI, B)

The positive answer requires the implementation of a diagnostic pathway comprising:

- the International Index of Erectile Function (IIEF-15* o IIEF-5);

- case history;

- physical examination;

- laboratory tests (testosterone, prolactin, TSH, PSA);

- an evaluation of vegetative cardiovascular tests can be useful.

* Depending on the score obtained, the IIEF-15 classifies ED in: severe $(<10)$, moderate (11-16), mild (17-26) and, absent (26-30). (VI, B)

Other tests are not generally required unless surgery is recommended. (VI, B)

Medical treatment recommends the use of PDE-5 inhibitors (sildenafil, vardenafil, tadalafil), taking into account the specific pharmacokinetic characteristics and, especially, the duration of their action ( $4 \mathrm{~h}$ for sildenafil and vardenafil, over $17 \mathrm{~h}$ for tadalafil). Weight loss, physical exercise and improved glycemic control can be useful. (VI, B)

Foot care

\section{Screening and prevention}

All patients with diabetes mellitus must undergo a complete examination of the foot at least once a year. Feet must be examined in high-risk patients on every visit. (VI, B)

Risk factors for diabetic foot must be defined at the time of screening. The subsequent follow up can be scheduled to suit the risk and presence of lesions. (VI, B)

All diabetics must be guaranteed an educational programme on the diabetic foot. (I, A) 
A diabetic foot care team should include doctors specialised in diabetic foot care, health-care professional qualified in the educational field and trained in diabetic foot care (podiatrist and/or nurses). (VI, B)

The elderly (age $>70$ years) require special attention, especially if they live alone, if they have a long-disease duration and if they have impaired vision and financial problems, since they have a higher risk of foot lesions. (III, B)

Specific healthcare pathways must be organised for the management of diabetic feet in diabetics who are either warded in long stay wards or are following a homecare programme. (VI, B)

Patients with feet at risk of lesions must be prescribed appropriate footwear and orthotic devices that reduce peak plantar pressure. (II, B)

\section{Foot examination}

The foot examination should include the evaluation of the case history of past ulcers and amputations, examination, palpation, evaluation of the perception of pressure (with the $10 \mathrm{~g}$ Semmes-Weinstein monofilament) and of vibration (with the tuning fork $128-\mathrm{Hz}$ or with the biothesiometer). (I, A)

Screening for peripheral arteriopathy should include a history for claudication, assessment of the pedal pulses and measurement of the ankle-brachial index (ABI). (III, B)

\section{Treatment}

High-risk diabetics, especially those with either past or present ulcers, require a multidisciplinary approach. (I, A)

Healthcare for patients with a foot lesion should be organised in three levels: first level (screening and diagnosis); second level (medication, minor surgery, off-load neuropathic plantar lesions); third level (distal, surgical and intraluminal revascularization procedures, orthopaedic surgery, both urgent and elective). (VI, B)

Urgent admission to hospital is recommended for patients presenting one of the following clinical pictures:

- critical ischemia;

- acute infection.

\section{(VI, B)}

Request the consultation of a multidisciplinary team expert in foot care within $24 \mathrm{~h}$ of evidence of either ulcers or a foot infection, to implement the following actions:

- urgently treat acute infections (cellulitis, gangrene, necrotizing fasciitis);

- appropriately manage the wound with debridement, surgical treatment of osteomyelitis, dressing;
- start systemic antibiotic therapy (often long term) for cellulitis or bone infections;

- off-load lesions;

- study and manage arterial failure;

- request radiological investigations (both conventional and MRI imaging), bone biopsy in case of suspected osteomyelitis;

- optimise glycemic control. (VI, B)

Patients with vascular disease and ulcers must be referred to distal, surgical and intraluminal revascularization procedures, both urgent and elective. (III, B)

For adequate pressure relief the use of a plaster or fibreglass off-loading cast is recommended in the management of neuropathic plantar ulcers without ischemia (TcPO2 > $30 \mathrm{mmHg}$ ). (II, B)

During the acute phase of Charcot foot, apply a stiff orthotic device and off-load the foot for 3-6 months to avoid deformity while awaiting recovery. (VI, B)

Do not resort to major amputation unless a detailed vascular evaluation has been performed and one or more of the following conditions is present:

- ischemic rest pain that cannot be managed with analgesics or revascularization;

- life-threatening infection that cannot be treated otherwise;

- a non-healing ulcer that does not heal and causes an higher burden than an amputation. (VI, B)

Systemic hyperbaric oxygen therapy is recommended to save the limb in the management of acute infections. (III, C)

Vacuum-assisted closure (VAC) therapy is recommended in the management of non vascular diabetic ulcers. (II, B)

Autologous cultured grafts reduce the healing time of neuropathic ulcers, especially when they are located on the dorsum of the foot. (II, B)

The prescription of orthotic devices (i.e. appropriate footwear and custom-made insoles) is recommended to prevent relapses in patients when ulcer has healed. (VI, B)

\section{Diabetes care in specific populations}

Diabetes care in children and adolescents

\section{Type 1 Diabetes}

Glycemic control All children and adolescents with diabetes mellitus must be followed by a multidisciplinary team of specialists from the time of diagnosis. (VI, B)

The choice of glycemic goals must be personalized in the various age groups, balancing the beneficial effect of 
achieving low HbA1c levels with the risk of hypoglycemia. Recommended HbA1c goals are $6.5-8.5 \%$ at $0-6$ years; $<7.5 \%$ at $6-12$ years; and $<7.5 \%$ in adolescents (age: $13-$ 19 years), if it can be reached without too many hypoglycemic episodes. (III, B)

Screening of associated autoimmune diseases (thyroid disorder and celiac disease) On diagnosis run the following tests: TSH, FT4; antithyroid antibodies, IgA, EMA or antitransglutaminase.

Yearly check TSH, antithyroid antibodies, EMA or antitransglutaminase. If EMA or antitransglutaminase are positive on two occasions, perform an intestinal biopsy for histological evidence of celiac disease. (VI, B)

Screening and management of chronic complications Nephropathy Yearly screening for microalbuminuria should be initiated at the age of 10 or after 5 years of disease duration. (III, B)

Once confirmed, persistent high levels of microalbuminuria must be treated with a titrated ACE-inhibitor, if possible, till microalbuminuria is normalized. (IV, B)

Hypertension Management of normal-high-pressure values (see below) must include, when appropriate, an intervention on diet and on physical exercise focused on weight control and on increased physical exercise. Pharmacological treatment should be started, if pressure goals are not achieved after 3-6 months of intervention on the lifestyle. (III, B)

ACE-inhibitors must be taken into account as initial treatment of hypertension. (III, B)

Dyslipidemia Prepubertal children: fasting lipid profile must be tested on all children aged $>2$ years on diagnosis of diabetes (after appropriate glycemic control has been reached) with a family history of hypercholesterolemia (total cholesterol $>240 \mathrm{mg} / \mathrm{dl}$ ), positive family history of cardiovascular events before the age of 55 or unknown family history. If the family history is negative, the first lipid screening should be performed at puberty $(>12$ years). If values are within acceptable risk levels (LDL cholesterol $<100 \mathrm{mg} / \mathrm{dl}$ ), the test should be repeated every 5 years. (III, B)

Pubertal children ( $>12$ years): the fasting lipid profile must be tested on diagnosis of diabetes (after appropriate glycemic control is achieved). If values are within acceptable risk levels (LDL cholesterol $<100 \mathrm{mg} / \mathrm{dl}$ ), the test must be repeated every 5 years. (III, B)

Treatment of dyslipidemia must be based on the fasting lipid profile (especially on LDL cholesterol) evaluated after appropriate glycemic control is achieved: the goal of treatment is a LDL cholesterol value $<100 \mathrm{mg} / \mathrm{dl}$. (III, B)

Initial treatment must envisage both optimizing glycemic control and medical nutrition therapy to reduce the diet's saturated fat content. (VI, B)

The addition of pharmacological agents is recommended when LDL cholesterol is $>160 \mathrm{mg} / \mathrm{dl}$ and in patients with LDL cholesterol 130-159 mg/dl, considering the cardiovascular risk profile, after nutrition therapy and lifestyle changes have failed. (III, B)

Retinopathy The first ophthalmological evaluation must be scheduled at the onset and, if it is normal, repeated when the child is 10 years old and has had diabetes for 3-5 years. (III, B)

A yearly follow-up is generally recommended after the age of 10 years. Less frequent controls can be deemed acceptable, if proposed by the ophthalmologist. (VI, B)

Diabetes care before and during pregnancy

All diabetic women of childbearing age must be informed about the need to achieve good metabolic control before conception, the risk of unscheduled pregnancy and the need to schedule conception with effective contraception methods. (VI, B)

Every diabetic woman who wishes to start a pregnancy must undergo screening and treatment for any complications (retinopathy, nephropathy, neuropathy, cardiovascular disease). (VI, B)

Glycemic control must be optimised before the conception. The therapeutic goal is normal or near normal HbA1c levels, allowing for at most a $1 \%$ deviation from the upper normal limit. (III, B)

Insulin therapy must be readily established in all women who fail to achieve the glycemic goal with nutritional therapy. Oral hypoglycemic agents must not be administered during pregnancy, due to the lack of enough data on the absence of teratogenous effects. (VI, B)

Rapid acting insulin analogues-aspart and lys-procan be either maintained or added to treatment during pregnancy; but data on the use of the rapid acting analogue glulisine during pregnancy is scarce; lastly, the use of delayed action analogues, which lack adequate safety data, is not recommended. (VI, B)

The use of ACE-inhibitors, ARBs and statins is not allowed during pregnancy: hence, these drugs must be discontinued before conception. (VI, B)

The glycemic goals that women with either gestational diabetes or pregestational diabetes (type 1 or type 2 ) must achieve during pregnancy are listed below:

- $\quad \leq 95 \mathrm{mg} / \mathrm{dl}$ fasting blood glucose; 
- $\quad \leq 140 \mathrm{mg} / \mathrm{dl} 1 \mathrm{~h}$ after meals;

- $\leq 120 \mathrm{mg} / \mathrm{dl} 2 \mathrm{~h}$ after meals. (VI, B)

Insulin treatment must be readily started in women with gestational diabetes, if glycemic goals are not achieved after 2 weeks of nutritional treatment. (VI, B)

Insulin patterns must be personalized in gestational diabetes: patterns envisaging either 1 or 2 injections can be implemented, but intensive insulin treatment can be necessary to reach optimal blood glucose levels. (VI, B)

Women with type 1 pregestational diabetes require multiple insulin delivery either subcutaneously or by CSII. Intensive insulin treatment is usually required to reach glycemic targets, even in type 2 pregestational diabetes. (I, A)

Women with diabetes during pregnancy must self-test glycemia at home (4-8 tests/day) with preprandial, postprandial ( $1 \mathrm{~h}$ after the meal) and nighttime tests. Simplified chessboard patterns can be used in gestational diabetes treated only with the nutritional therapy. $(\mathbf{V}, \mathbf{B})$

Ketosis must be avoided during pregnancy; hence, daily ketonuria testing on awakening is useful. $(\mathbf{V}, \mathbf{B})$

Nutrition treatment during pregnancy must be personalized, taking into account both the diet habit of diabetic women and the BMI before the pregnancy. The goals are appropriate maternal and foetal nutrition, appropriate calorie, vitamin and mineral intake and optimal glycemic control when there is no ketonuria. (VI, B)

Diabetes care in the elderly

\section{Glycemic control}

Glycemic goals should be individualized in elderly diabetics. If general conditions are good, HbAlc levels can be $6.5-7.5 \%$. (VI, B)

In the frail elderly (with complications, dementia, multiple diseases, a high risk of hypoglycemia and, in whom the risk of intensive glycemic control exceeds expected benefits) a less restrictive goal is recommended with HbA1c levels between 7.5 and $8.5 \%$. (VI, B)

In elderly diabetics the self-monitoring pattern should be designed to suit the degree of self-sufficiency; hence, individual functional, affective and cognitive capacities. The pattern must be based on planned glycemic and $\mathrm{HbA1c}$ goals, on the real feasibility of changing treatment and on the risk of hypoglycemia. (VI, B)

If oral hypoglycemic agents are prescribed in the elderly, chlorpropamide and glibenclamide are not recommended. $(\mathbf{V}, \mathbf{B})$

In elderly diabetics with serum creatinine $\geq 1.5 \mathrm{mg} / \mathrm{dl}$ $(\geq 1.4 \mathrm{mg} / \mathrm{dl}$ in women) or creatinine clearance levels indicating impaired kidney function metformin is not recommended, due to the higher risk of lactic acidosis. (IV, B)
In elderly diabetics treated with metformin, serum creatinine should be tested at least once a year and at every dosage increase. Creatinine clearance should be tested in subjects aged $\geq 80$ years or presenting a reduced muscular mass. (VI, C)

\section{Cardiovascular risk and pharmacological treatment}

The cardiovascular risk must be evaluated in all elderly diabetics. (VI, B)

Dyslipidemia in elderly diabetics must be corrected, compatibly with an overall evaluation of the patient's health condition. (II, B)

If an elderly diabetic has LDL cholesterol $\geq 130 \mathrm{mg} / \mathrm{dl}$, he needs both pharmacological therapy and lifestyle changes. The lipid profile must then be retested at least once a year. $(\mathbf{I}, \mathbf{A})$

In elderly diabetics requiring antihypertensive pharmacological therapy, the treatment goal must be blood pressure $<140 / 80$, if it is well tolerated. A further drop in blood pressure $(<130 / 80)$ can involve an additional advantage. (I, A)

Considering that elderly subjects may poorly tolerate a pressure reduction (especially in case of past episodes of syncope, falls and orthostatic hypotension), antihypertensive treatment should be gradually established and titrated. (VI, B)

In elderly diabetics treated with ACE-inhibitors or sartans, kidney function and serum $\mathrm{K}$ should be tested within 1-2 weeks of the treatment start, whenever the dosage is increased and, anyhow, at least once a year. (VI, B)

Elderly diabetics treated with thiazides or loop diuretics require blood sodium and potassium testing within 1-2 weeks after the start of treatment, whenever the dosage is increased and, anyhow, at least once a year. (VI, B)

\section{Functional evaluation}

Elderly patients with type 2 diabetes require a geriatric multidimensional evaluation and an evaluation of geriatric syndromes. (VI, B)

The evaluation must include global/physical, cognitive and affective tests. (VI, B)

The functional evaluation must be completed with the assessment of comorbidities and nutritional status. (VI, B)

The elderly diabetic possibility of performing physical exercise should be periodically evaluated; he must be informed about the beneficial effects of exercise and the resources available to increase the degree of exercise. (VI, B)

Food intake, nutritional status and hydration should be periodically evaluated in elderly diabetics; they should be provided with nutrition therapy instructions that are appropriate for their social, economic and cultural 
condition, advice on diet contents and the potential advantages of body weight loss. The risk of calorie-protein malnutrition, which is rather frequent in the elderly, must always be evaluated. (VI, B)

Elderly diabetics have a higher risk of depression; hence the need for special focus to recognize symptoms suggesting this diagnosis, both during an initial evaluation and in case of a worsened clinical condition that cannot otherwise be justified. (III, C)

Elderly diabetics should be invited to keep an updated record of the drugs taken, which they must show their attending physician. (VI, C)

An elderly diabetic attending physician should take into account the possible presence of cognitive decline, both during the initial evaluation and when there is an otherwise unjustified decline in the clinical condition with, for instance, an increased difficulty to ensure safe care. (VI, C)

Yearly screening of the elderly diabetic should include the search for symptoms of incontinence. (VI, C)

The elderly diabetic should be questioned about any episodes of falls. In this case, the causes (i.e. drugs, environmental factors, etc.) will be investigated. (VI, C)

During the initial evaluation, the elderly diabetic should be questioned on the possible presence of chronic pain. (VI, C)

Every long stay ward admitting diabetic patients should have an established diabetes care plan or protocol that is subject to regular reviews. (VI, B)

\section{Diabetes care in specific frameworks}

Diabetes care in hospital

The diagnosis of diabetes mellitus must be clearly entered in the case records of all diabetic patients who are admitted to hospital. (VI, B)

If hyperglycemia is occasionally found during hospitalization, HbA1c testing should be performed to identify a condition of undiagnosed diabetes. $(\mathbf{V}, \mathbf{B})$

Capillary blood glucose must be monitored in all diabetics admitted to hospital and the results must be entered in the case records to ensure their access to all attending team members. (VI, B)

A treatment plan for hypoglycemia must be defined for every patient. Episodes occurring during hospitalization must be recorded in the case records. (VI, B)

Admission is not the most appropriate time to establish an organic educational program centered on diabetes. However, the diabetic must be instructed on some basic aspects-such as insulin injection and self-testing of blood glucose-before discharge. (VI, B)
Undiagnosed diabetics presenting hyperglycemia on admission to hospital must be referred to the competent Diabetes Unit. (VI, B)

\section{Glycemic goals}

During hospitalization, glycemic goals can be differentiated to suit the various clinical conditions:

- critical patients: blood glucose levels near $110 \mathrm{mg} / \mathrm{dl}$ and in any case $<180 \mathrm{mg} / \mathrm{dl}$; (II, B)

- non critical patients: preprandial blood glucose levels near 90-130 mg/dl, postprandial $<180 \mathrm{mg} / \mathrm{dl}$. (VI, B)

Glycemic goals should be gradually achieved in some clinical conditions presenting a high risk of hypoglycemia. (VI, B)

\section{Treatment}

The use of the main oral hypoglycemic drugs (secretagogues, biguanides, thiazolidinediones) has considerable limits in the hospital framework. Insulin delivery is, hence, the choice treatment for non stabilized hospitalized diabetic patients. (VI, B)

Subcutaneous insulin treatment must preferably follow a scheduled scheme, which is frequently adapted to suit recorded blood glucose levels. This pattern can be integrated with a correcting algorithm based on blood sugar testing at the time of the injection. Insulin delivery only "when required" (sliding scale) is not recommended due to its poor efficacy. (IV, B)

In critical patients and/or those who are unable to take food by mouth, during the perioperative period and in conditions of acute metabolic instability, insulin therapy must be delivered as continuous venous infusion, applying algorithms based on frequent blood glucose testing. (VI, B)

Non critical patients who are expert in both self-delivery of insulin and blood glucose self-testing can be authorised to continue self management even during hospitalisation, agreeing on the specific modes with the attending healthcare team. (VI, B)

In patients already under treatment with an insulin pump (CSII), it can be useful to maintain this method even during hospitalization, as long as it can be correctly managed in the specific clinical situation. (VI, B)

Diabetes care at school and in day care centres

The diabetes team must draft a personalized care plan for the diabetic student with parents/guardians. (VI, B) 
An appropriate number of schoolteachers or other employees should be trained in procedures to be implemented for hypoglycemic episodes. These people must not necessarily be healthcare professionals. (VI, B)

Diabetic students must have instant access to whatever they need for diabetes treatment at all times and under supervision if it is necessary. (VI, B)

The student must be able to test blood glucose in the classroom and to treat hypoglycemia either in the classroom or wherever he is for schooling activities, as envisaged by his care plan. (VI, B)

Diabetes care in diabetes educational camps

Every participant in an educational camp must have a standardized case record filled out by both the family and the attending diabetologist. (VI, B)

Medical healthcare staff must be guided by an expert in the management of type 1 and type 2 diabetes and must comprise educators and nurses specialized in diabetes, besides dieticians skilled in diabetes. (VI, B)

All camp personnel-doctors, nurses, nutritionists and voluntary workers-must undergo a basic training test to ensure the appropriate working mode with children. (VI, B)

Diabetes in houses of correction

At the time of access to the house of correction, diabetics should promptly have a complete case history taken and undergo a complete examination on the part of healthcare personnel. (VI, B)

Patients with type 1 diabetes must be identified due to their high risk of diabetic ketoacidosis. Insulin treated patients should have capillary blood glucose tested within 1-2 $\mathrm{h}$ after arrival. (VI, B)

Pharmacological treatment must be continued without interruption. (VI, B)

The penitentiary doctor should establish every patient's calorie requirement and diet composition. (VI, B)

Menus served must be balanced and based on healthy Italian diet recommendations. (VI, B)

Capillary blood glucose testing must be organized following formal protocols well defined to suit the type of diabetes, treatment and diet. (VI, B)

If the patient is imprisoned in a penitentiary institute that lacks continuous nursing service, he should be moved to a second level penitentiary institute to appropriately face diabetes-related clinical needs and insulin delivery, if required. (VI, B)

Prisoners under treatment with oral hypoglycemic agents and/or insulin with a tendency to self harm and those who have psychiatric disorders must be ensured special attention. (VI, B)

The patient must be provided with a source of rapid absorption sugars to be taken at the first signs of a hypoglycemic crisis. (VI, B)

Regular continuous physical exercise must be encouraged (at least 30 min.) 3-4 days a week; a daily activity plan to be performed during airing time can be agreed with the patient when there are no appropriate facilities. (VI, B)

Procedural protocols must be processed and spread to ensure the entire healthcare staff appropriate knowledge on the management of metabolic emergencies (i.e. hypoglycemia and hyperglycemia); moreover, the diabetic patient must be provided with appropriate education. (VI, B)

Reference facilities must be found either inside or outside the main clinical centers of penitentiaries for diagnosis and periodical staging of chronic complications and for the management of diabetic emergencies. (VI, B)

If a diabetic is transferred from one house of correction to another, a summary medical report must be filled out to accompany the patient on the journey. (VI, B)

Medications and drugs required to treat diabetes must accompany the patient on the journey. (VI, B)

The discharge plan must be established in appropriate advance to ensure care continuity and, in case of release from prison, to facilitate the patient's admission to external healthcare facilities. (VI, B)

\section{Appendix: Indicators}

Introduction

\section{Measurement, analysis and improvement}

The measurement of professional performance and the subsequent feedback of information is essential in facilitating knowledge building and ongoing improvements in healthcare.

The growing worldwide interest in indicators mirrors the need to measure processes and healthcare outcomes. Hence, diabetic unit must plan and implement monitoring, measurement and analysis actions required to prove product compliance with the standards of the best current scientific knowledge and, to steadily improve the efficacy of healthcare activities.

The diabetic unit should overcome the concept of oneto-one duties, of attention focused on the single patient and, hence, on measurement of the efficacy of personal actions on individual parameters to build an information system designed to systematically collect individual data with the aim to measure the success of global performance. The goal is to highlight problems in an attempt to change 
Table 6 Quality measurement principles

ISO 9001:2000

8.2.3 Monitoring and measurement of processes

Monitor and measure the performance of the processes that make up the QMS. Compare these actual results to the planned results. Take corrective action to make sure the product or service meets requirements

\section{ISO 9001:2000}

\subsubsection{Monitoring and measurement of products}

During the production process, monitor and measure the product to assess if requirements are met. Keep records showing:

The product meets acceptance criteria.

The name of the person who authorized release of the product.

The product has proceeded through all of the planned process steps, including all planned verifications.

the clinical, therapeutic, management and economic outcome (see Table 6).

Indicators are variables that describe the complex phenomena of healthcare to facilitate decisions concerning either implementing or maintaining changes.

An indicator requires quality attributes, in fact, it must:

measure important aspects of healthcare quality;

measure in a valid, precise, accurate, reproducible manner; be speedily measurable in the framework of available resources;

be able to direct decisions;

be able to differentiate the various conditions;

obtain consensus regarding its meaning and mode of use.

From: Joint Commission on Accreditation of Healthcare Organizations. Characteristics of indicators in primer on indicator development and applications. Oakbrook Terrace (IL) 1990 .

Hence, the choice of indicators that can meet all quality requirements, either entirely or almost entirely.

Type of indicators used:

1. process

2. intermediate result

3. final result.

Note: measurement methods must ensure that products comply with the current standards and expectations of reference subjects' privileged indicators obtained from data collected during daily activity management. The AMD data file, a tool currently accessible in Italy to those who either use compatible electronic case records (most of them) or wish to do so, was referred in this perspective. The complete list of indicators is published at http://www.aemmedi.it/, http://www.siditalia.it/. 